\title{
Design Study of an Underground Detector for Measurements of the Differential Muon Flux
}

\author{
Bogdan Mitrica \\ Horia Hulubei National Institute of Physics and Nuclear Engineering-IFIN HH, P.O. Box MG-6, 077125 Magurele, Romania
}

Correspondence should be addressed to Bogdan Mitrica; mitrica@nipne.ro

Received 3 July 2013; Accepted 9 July 2013

Academic Editor: Carlo Cattani

Copyright (C) 2013 Bogdan Mitrica. This is an open access article distributed under the Creative Commons Attribution License, which permits unrestricted use, distribution, and reproduction in any medium, provided the original work is properly cited.

Since 2006 an underground laboratory is in operation in Unirea salt mine from Slanic Prahova Romania. A new rotatable detector for measurements of the directional variation of the muon flux has been designed and will be put in operation at the end of 2013 . The detector will be used to investigate the possible presence of unknown cavities in the salt ore. Preliminary muon flux measurements performed in the underground of Slanic Prahova salt mine show an important variation of the flux with the thickness of the rock but indicate also that more precise data are necessary. Based on that, a modern detector using 4 layers of plastic scintillators bars has been designed. The detector is installed on a rotatable and mobile frame which allows precise directional measurements of the muon flux on different locations in the mine. In order to investigate the performances of the detector, detailed Monte Carlo simulations have been performed using several codes available on the market. The simulations show that the detector can be used for measurements of the differential flux of cosmic ray muons and for the detection of hidden cavities in the ore.

\section{Introduction}

During the last decade, a number of events implying collapsed cavities have been observed all over the world [1, 2]. These events illustrate the major risk represented by the presence of unknown cavities in different ores, especially on areas with historical mining activity. Starting from this problem waiting to be solved, an underground muon detector was built and will be put in operation in Slanic Prahova salt mine.

Since the detection of hidden cavities is very important and actual especially for the locations with mining activity, a number of methods based on different techniques are available in the market. Those methods are based on ultrasounds (US), X-ray, or even gamma ray detection. Several companies (e.g., http://www.socon.info/) over the world are presently performing this kind of measurements on request, but the results are affected by secondary effects and experimental uncertainties. The ultrasounds could affect the stability of the rocks, and the gamma and X-rays could produce important changes in the radioactive background of the investigated area. Another problem of these methods is represented by the relative high cost of the detectors and of the services.
Difficulties regarding the environmental problems are also influencing the prices and the feasibility of the methods.

We propose an innovative and $100 \%$ safe method to detect the hidden cavities based on the measurements of the muons from the secondary cosmic radiation, which is the most important source of natural radiation.

1.1. The Cosmic Rays. From 1912, when Victor Hess has discovered the cosmic rays [3], until the present days, an important advance was made in this field of research. Shaded for a period of time by the parallel development of particle accelerators, an increasing interest is granted lately to cosmic rays, largely due to the higher energetic possibilities and a much lower cost of the infrastructure. The particle accelerators make it possible to obtain and investigate particles of very high energy [4], but that is still very far from the energies observed in the cosmic rays spectrum (up to $10^{20} \mathrm{eV}$ ) [5]. The primary cosmic rays consist of protons (90\%), He nuclei (7\%), and other nuclei (3\%). The energy spectrum covers more than 21 orders of magnitude. The measurements of the spectrum of the primary cosmic rays could be performed by direct measurements only for energies $<10^{14} \mathrm{eV}$. For higher energy, up to $10^{20} \mathrm{eV}$, where 
the flux is lower than 1 particle $/ \mathrm{km}^{2}$ century, the spectrum could be measured only by indirect experiments that cover large areas at the surface of the Earth [6-9]. There are a few open problems addressed by the researchers regarding the cosmic rays, such as the investigation of the hadronic interaction models at high energies [10], where no data from the accelerators are available, or the origin of cosmic ray particles that are still far from a clear solution. Many simulation codes and computational models are available on the market [11-13], making possible detailed calculations for different processes at high and low energies.

1.2. The Muons. Interacting with the atmosphere of the Earth, cosmic rays break into numerous components, also named the secondary cosmic radiation, generating Extended air showers that propagate until they reach the ground. Excepting the photons and neutrinos, muons are the most abundant component of the secondary cosmic radiation at the sea level. As leptons, muons are less affected by hadronic interactions, also they show reduced electromagnetic interactions and hence interact weakly with matter. They penetrate large thicknesses of matter before they are stopped and decay, and they are historically known as p̈enetrating componentsöf secondary cosmic rays, even detectable in deep underground sites. At sea level the inclusive flux is about 120 muonss $^{-1}$. $\mathrm{m}^{-2} \cdot \mathrm{sr}^{-1}$ and comprises about $80 \%$ of the total atmospheric flux of secondary charged cosmic ray particles. Consequently much more information could be given by muons than on any other component, such as information on high energy processes in the atmosphere or on the primary radiation, in particular on its spectrum and composition. It is evident that the flux of atmospheric muons is a quantity of high interest and information which characterizes the site of a nuclear physics laboratory which is planned for far-reaching investigations. Another reason for measuring the flux of the atmospheric muons in the underground arises from the practical necessity of information on the cosmic radiation background for different sites, for experiments like $[14,15]$, and so forth.

In the near past, the idea to use to use muons for the detection of hidden cavities has been used in the search for hidden chambers in ancient pyramids from Giza [16] or the Aztec Pyramid of the Sun at Teotihuacan [17]. Since these experiments reported interesting results, one can conclude that the cosmic ray muons could be used for the purpose of this project with high expectation. In the present days, some attempts to use muons for geophysical application have been done [18], but no device for the detection of hidden cavities is available in the market. We propose to fill this empty position and will bring a new and modern solution for the security of people over the world. The method will be further extended for new application as geophysical data or speleology.

The idea of using cosmic ray muons to improve the quality of life has great potential but is underestimated and largely unexplored. During the last years some preliminary results have been obtained in IFIN-HH for measurements of the muon flux in underground of Unirea mine, at sea level, and at ground level at different elevations.
In this paper we report about the design and construction of an underground muon detector that could be used to detect unknown cavities in different areas with mining activity. Detailed Monte Carlo simulation using MUSIC [19] and CORSIKA [11] codes has been used in order to establish the performances of this new device. The simulations are controlled by muon flux measurements performed with the mobile muon detector of IFIN-HH [20,21].

\section{Measurements of the Muon Flux in the Underground}

Measurements of the cosmic ray muon flux have been performed in the underground of Slanic Prahova salt mine using the mobile detector of IFIN-HH. The detector has been designed for underground and ground level measurements of the total muon intensity.

Preliminary muon flux measurements have been performed using the mobile detector of IFIN-HH [20, 21]. The detector consists of 2 modules each one having 4 scintillator plates of $25 \times 100 \times 10 \mathrm{~cm}$ covering a surface of $1 \mathrm{~m}^{2}$. The system is mounted on a van which allows a large mobility on ground level or in the underground. The signal from the each PMT is read out independently. Further, the four signals from each plate are ored, and then the results from both modules are put in coincidence. Directional measurements combining 2 plates from both modules could be performed in order to see the angular variation of the flux. Due to the large angular acceptance, no precise directional variation of the muon flux is possible.

The angular acceptance of the detector was determined by Monte Carlo simulation using GEANT4 [22] and CORSIKA [11] codes. A correction factor of 1.56 should be applied to the raw data in the case of the detector mounted on the van and 1.11 in the case of the plates placed one on top of each other.

2.1. The Slanic Prahova Salt Ore. The salt ore from Slanic Prahova consists of a lens of $500 \mathrm{~m}$ thickness, few kilometers long and wide (see Figure 1). The salt is extracted from the Slanic mine continuously since ancient times, and due to this fact, many galleries (i.e., shaped caverns) are already excavated.

Presently few mines are well known in Slanic Prahova salt ore. Some of them are used for touristic activities (Unirea and Mihai), one of them (Cantacuzino) is still used to extract salt, and others (Victoria and Carol) are closed for security reasons. Some other ancient cavities close to the surface are used also for touristic activities. The existing cavities will be used to calibrate the detector in the underground.

Since 2006 a modern underground laboratory ( $\mu$ Bqlab) $[23,24]$ for low background measurements was developed by IFIN-HH in the Unirea mine from Slanic Prahova. The location of the laboratory is indicated in Figure 1. During 2012 the laboratory has been upgraded and new scientific infrastructures have been achieved. One of them is represented by a new rotatable mobile detector for measurements of the directional variation of the muon flux. 


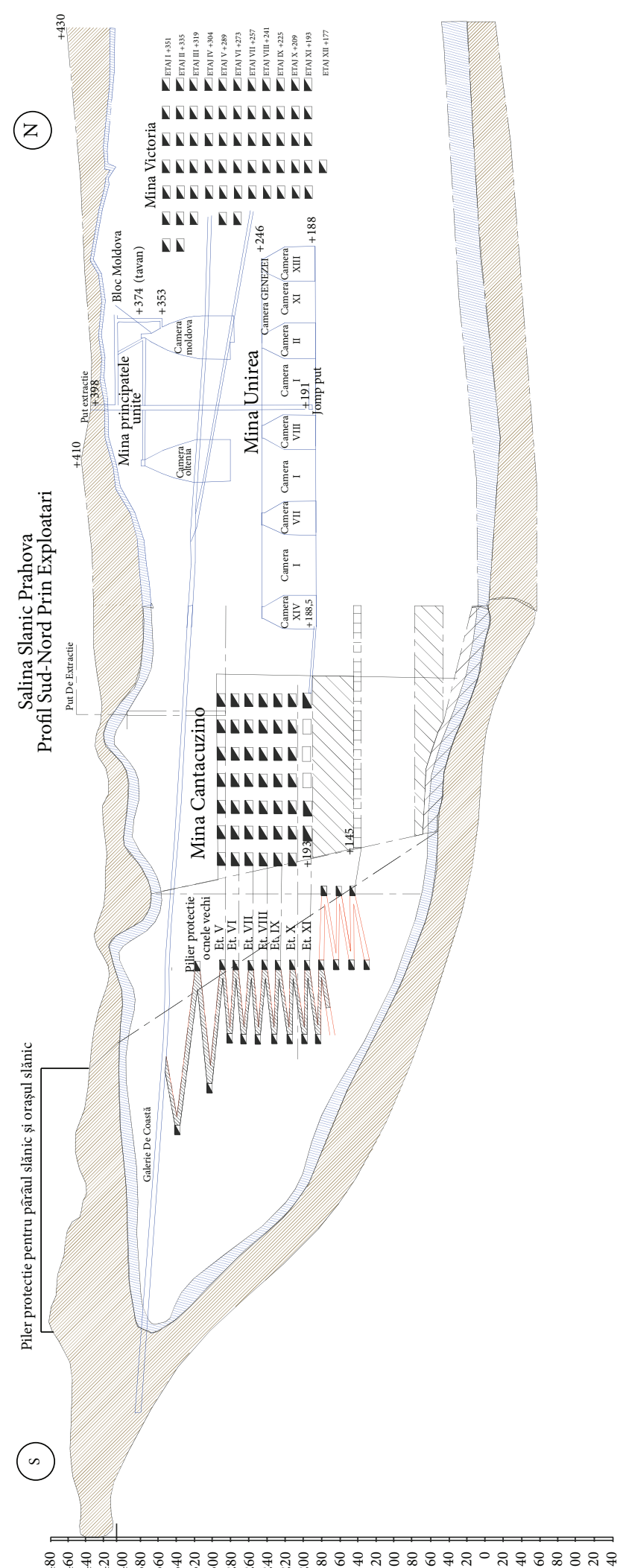

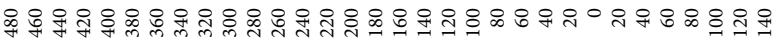

FIGURE 1: Artistic view of the salt ore of Slanic.
The "Unirea" mine is characterized by temperature: 12.0$13.0^{\circ} \mathrm{C}$, humidity: $65-70 \%$, excavated volume: 2.9 million $\mathrm{m}^{3}$, floor area: $70000 \mathrm{~m}^{2}$, average high: $52-57 \mathrm{~m}$, aerosols $<10 \mu \mathrm{m}$ : $2 \cdot 10^{8} \mathrm{part} / \mathrm{m}^{3}$, and distance between walls: $32-36 \mathrm{~m}$, existing infrastructure: electricity, roads, railway, elevator, phone, Internet, and GSM networks (also inside the galleries). Slanic Prahova is also the Romanian proposal for LAGUNA-LBNO [14] experiment which is intent to build a huge detector with an active volume around $100.000 \mathrm{~m}^{3}$. From the Slanic site a huge volume of material has been already excavated, but the shallow depth could induce a problem. Following [14], for the GLACIER [25] experiment, the Slanic mine could be a feasible location as for this technique a depth of only 600 mwe is necessary, comparable with the one of Unirea mine.

2.2. Preliminary Muon Flux Measurements. The muon flux has been measured in Unirea, Mihai, and Cantacuzino mines at different levels, and some of the results was previously reported in $[20,21]$. New measurements in the main and in a small cavity of Mihai mine have been performed. The results are presented in Table 1. The theoretical depth of each mine has been determined taking in to account the presence of a standard rock hill above the entrance in the mine. The real depth has been obtained considering the geometrical characteristics of each cavity. Unfortunately, due to the nonuniform shape of the cavities, the results need to be corrected and will be estimated based on directional variation of the muon flux. The results are compared with Monte Carlo simulations based on MUSIC code [19].

From Table 1 one can observe that the muon flux is quite sensitive to the variation of the rock thickness. Unfortunately, the direction of the muons and the location of different caverns are not easy to be determined. For more precise measurements, it is mandatory to have a detector which allows to establish the direction of the incoming muon and which permits to establish the presence and the location of possible unknown cavities.

\section{The New Rotatable Detector}

In order to perform precise measurements of the directional variation of the muon flux, a new detector was designed and constructed; the measurements are planned to start at the Autumn of 2013. The detector consists in 160 plastic scintillator bars type UPS 923A of $26 \times 1000 \times 100 \mathrm{~mm}$ (see Figure 2) produced by AMCRYS Ltd. Ukraine [26]. Each one has a stripe right on the middle where an optical fiber will transfer the optical pulse to the PMT [27].

The scintillator bars are mounted in 4 boxes ( 40 pcs in each box) and the signal from each one is transferred to one of the two micro-PMTs of type R11265-112-M64 [28] (see Figure 3) mounted on each box (see Figure 4).

The 4 boxes are mounted 2 by 2 at top of each other and orientated in cross. Then each group of 2 is separated by the other one by a distance of $0.8 \mathrm{~m}$, and the entire system is placed on a rotatable and mobile frame (see Figure 5). The direction of each incident muon is determined using the $x$ and $y$ positions given by the signals from each scintillator bar from both layers of 2 . 
TABLE 1: The muon flux data obtained in underground measurements.

\begin{tabular}{|c|c|c|c|c|c|}
\hline Location & $\begin{array}{l}\text { Theoretical depth } \\
(\mathrm{m})\end{array}$ & $\begin{array}{l}\text { Muon flux for the } \\
\text { theoretical depth } \\
\left(\mathrm{m}^{-2} \mathrm{~s}^{-1}\right)\end{array}$ & $\begin{array}{l}\text { Real depth } \\
\quad(\mathrm{m})\end{array}$ & $\begin{array}{l}\text { Muon flux for } \\
\text { the real depth } \\
\left(\mathrm{m}^{-2} \mathrm{~s}^{-1}\right)\end{array}$ & $\begin{array}{l}\text { Muon flux } \\
\left(\mathrm{m}^{-2} \mathrm{~s}^{-1}\right)\end{array}$ \\
\hline Unirea & 297 & 0.051 & 170 & 0.18 & 0.18 \\
\hline Mihai-main cavity & 204 & 0.12 & 90 & 0.51 & 0.52 \\
\hline Mihai_small cavity & 204 & 0.12 & 125 & 0.32 & 0.33 \\
\hline Cantacuzino mine-level 5 & 173 & 0.17 & 165 & 0.19 & \\
\hline Cantacuzino mine-level 6 & 189 & 0.15 & 173 & 0.17 & \\
\hline Cantacuzino mine-level 7 & 205 & 0.12 & 181 & 0.16 & \\
\hline Cantacuzino mine-level 8 & 221 & 0.11 & 189 & 0.15 & 0.19 \\
\hline Cantacuzino mine-level 9 & 237 & 0.10 & 197 & 0.13 & \\
\hline Cantacuzino mine-level 10 & 253 & 0.08 & 205 & 0.12 & \\
\hline Cantacuzino mine-level 11 & 269 & 0.06 & 213 & 0.11 & 0.09 \\
\hline
\end{tabular}

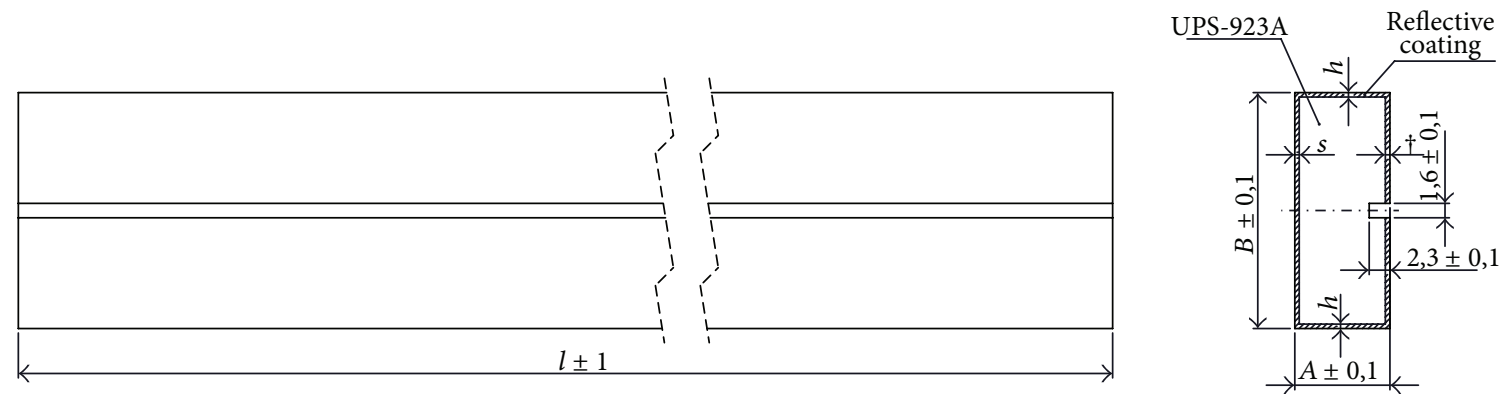

FIgURE 2: The scintillator bar [26].

The information from PMT is read out by modular electronic devices produced by CAEN [29] and National Instruments [30]. The detector permits directional measurements of the muon flux up to $45^{\circ}$ zenith angle. We estimate that for the Slanic Prahova salt ore a realistic time of data acquisition will be from 2 to 3 days for a zenith angle from 0 to $10^{\circ}$ up to 3 months for $45^{\circ}$.

\section{Simulation of the Detector's Performances}

For the studies regarding the detector performances, detailed Monte Carlo simulation is necessary. The directional variation of the muon flux in the underground should be accurately simulated in order to investigate the detector response. For this task, several options represented by computational codes are available. Some of them (e.g., GEANT4 [31] or FLUKA $[32,33])$ can give more precise results but request a longer running time. Instead, the MUSIC code could give realistic results in a shorter time.

MUon SImulation Code (MUSIC) [19] is a computational model for 3-dimensional simulations of the muon propagation through rock. It takes into account energy losses of muons by pair production, inelastic scattering, bremsstrahlung, ionization and the angular deflection by multiple scattering. The program uses the standard CERN library routines and random number generators. The code has been originally designed for simulation of the muon flux at high depths (more than 500 m.w.e.). Since the necessity of the detection of hidden cavities requires an estimation of the muon flux at low depths, in this paper, the code has been modified in order to overlap its limits. For this task, it is important to know the muon flux at ground level. To estimate the muon flux at ground level, one can choose from several options represented by semianalytical formulas or detailed Monte Carlo simulation.

The muon flux is defined as the number of muons transversing a horizontal element of area per unit of time [5]:

$$
J_{\mu}=\frac{d N}{d t \cdot d A \cdot d \Omega \cdot d P} \quad\left(\mathrm{~cm}^{-2} \cdot \mathrm{s}^{-1} \cdot \mathrm{sr}^{-1} \cdot(\mathrm{GeV} / \mathrm{c})^{-1}\right) .
$$

There are several empirical approximations describing the fluxes by analytical expressions like power-law distributions (see Grieder [5]). Recent approaches by Gaisser [34] display explicitly the dependence on primary energy but with complicated mathematical procedures and valid only for muon energies above $10 \mathrm{GeV}$. This holds also for the simplification given in Gaisser's Book [35]

$$
\begin{aligned}
\phi_{\mu}= & \frac{0.14}{\mathrm{~cm}^{2} \cdot \mathrm{s} \cdot \mathrm{sr} \cdot \mathrm{GeV}} \cdot(E / \mathrm{GeV})^{-2.7} \\
& \times\left[\frac{1}{1+(E \cdot \cos \theta / 110 \mathrm{GeV})}+\frac{0.37}{1+(E \cdot \cos \theta / 760 \mathrm{GeV})}\right]
\end{aligned}
$$




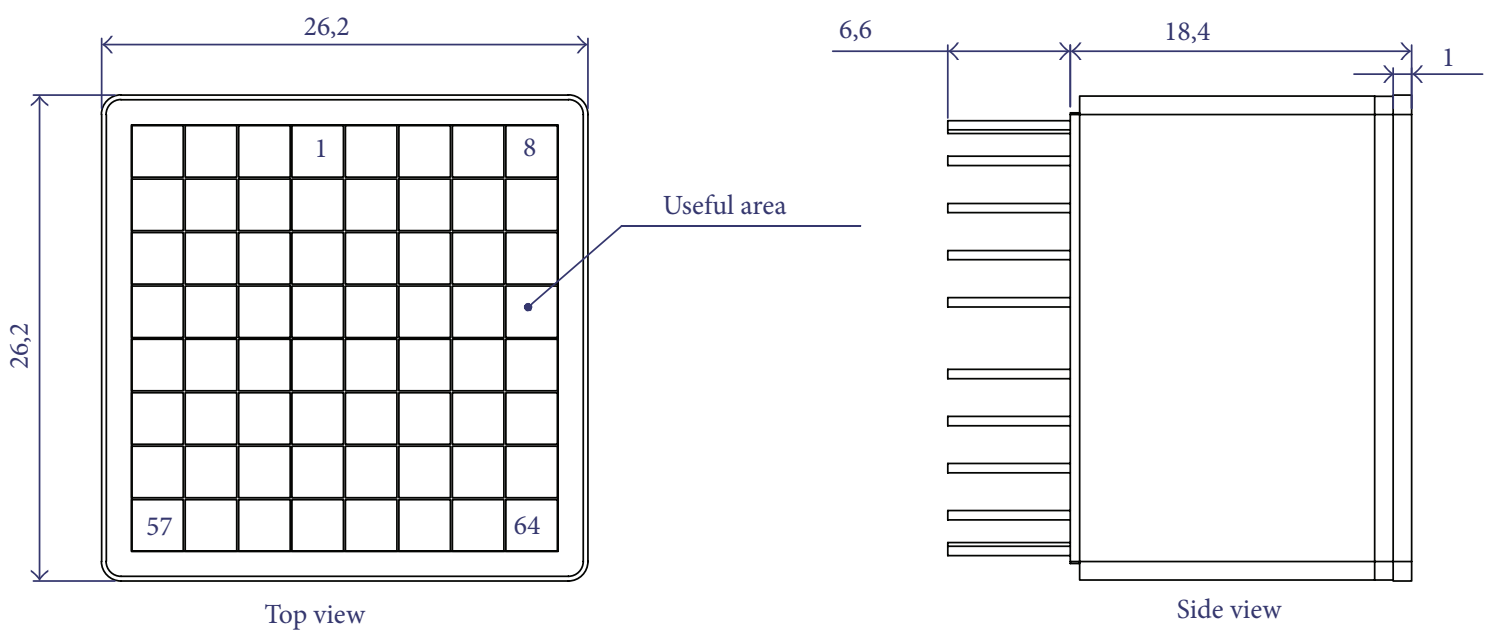

FIgURE 3: The microphotomultiplier tube [28].

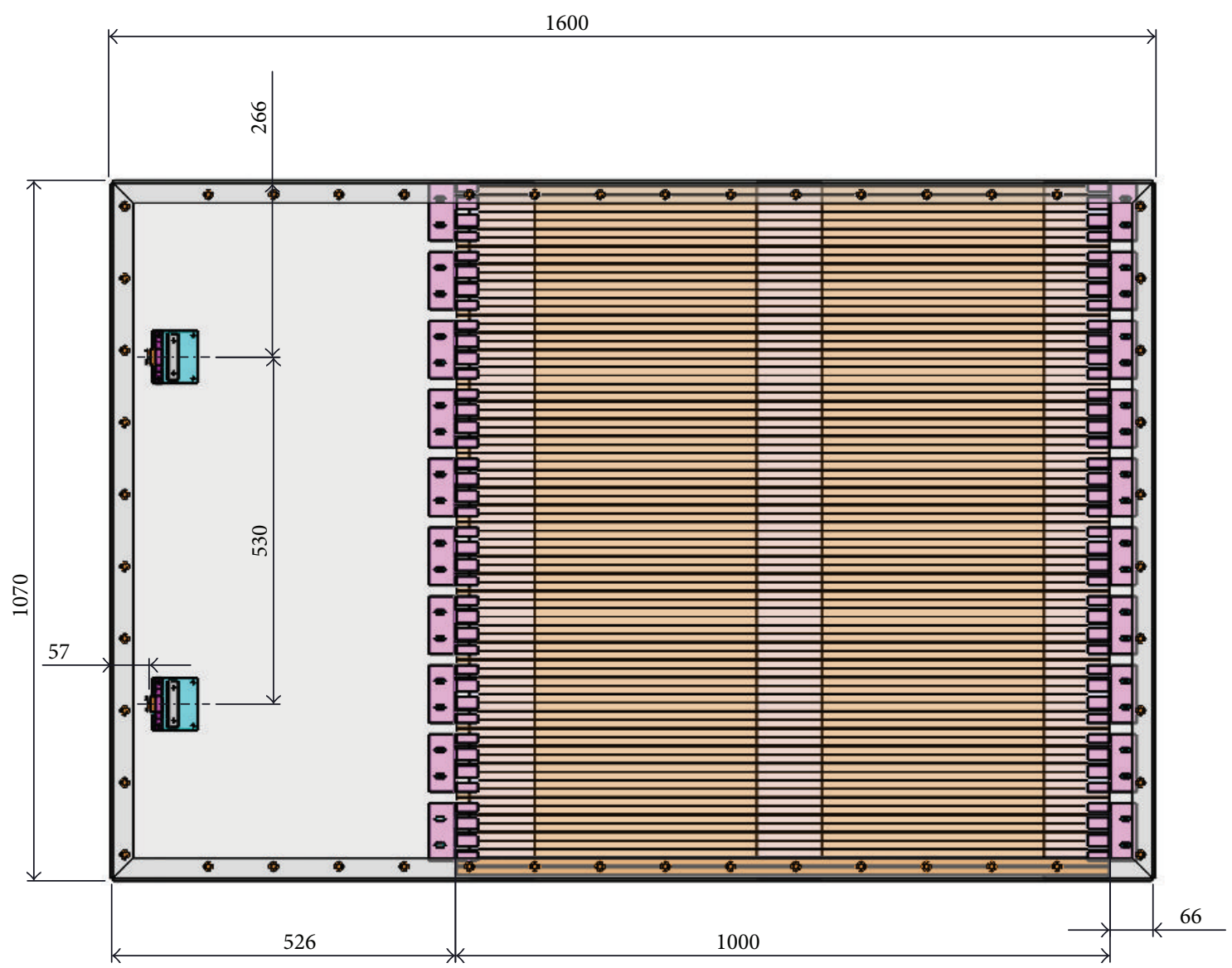

FIgURE 4: The detection module.

used, for example, by Unger et al. [36]. In Figure 6 this formula is compared with the results of the Monte Carlo simulations, displaying the disagreement in particular at lower energies.

The approach by Judge and Nash [37] uses as input the production spectra of parent pions and kaons and calculates the flux resulting from pion and kaon decay by

$$
\begin{aligned}
& D_{\pi}\left(E_{\mu}, \theta\right)=\frac{A_{\pi} \cdot W_{\mu} \cdot E_{\pi}^{-\gamma_{\pi}} \cdot H_{\pi}}{E_{\pi} \cdot \cos \theta+H_{\pi}}, \\
& D_{k}\left(E_{\mu}, \theta\right)=\frac{A_{k} \cdot W_{\mu} \cdot E_{k}^{-\gamma_{k}} \cdot H_{k}}{E_{k} \cdot \cos \theta+H_{k}} .
\end{aligned}
$$

There $H_{\pi, k}$ and $H_{\mu}$ are parameters accounting for the propagation of the particles in the atmosphere, and $\theta$ is the 


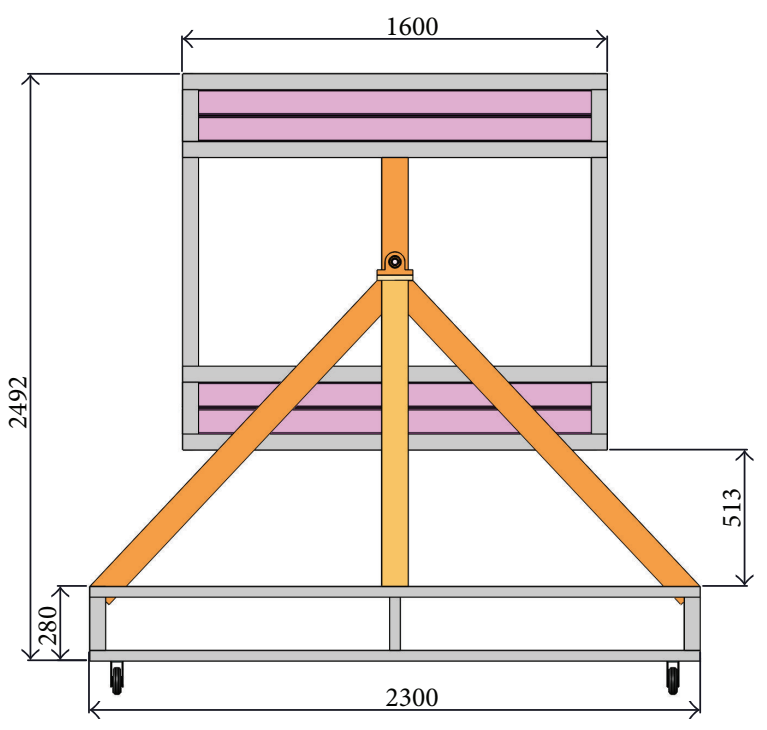

(a)

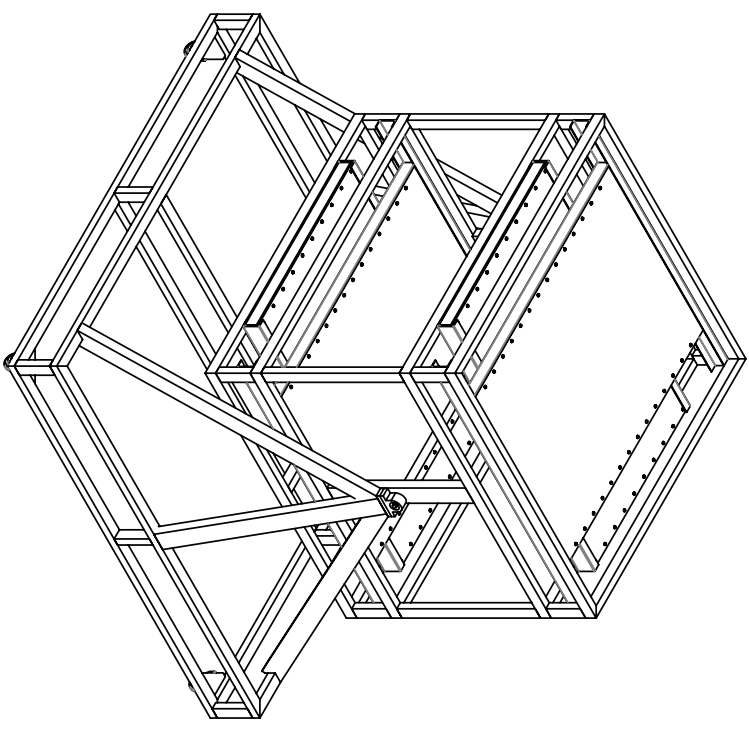

(b)

Figure 5: The rotatable detector.

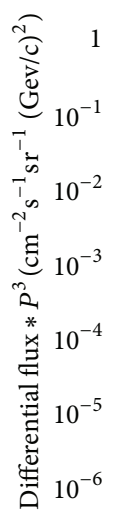

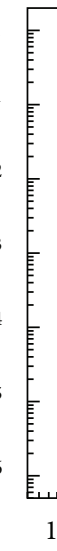

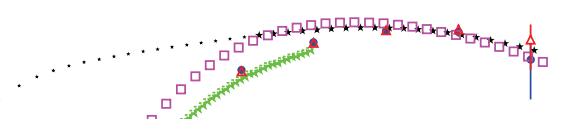

- CORSIKA simulations for Bucharest

* Semianalytical formula of Gaisser

- Semianalytical formula of Nash

Experimental data BESS

- WILLI (preliminary)

Figure 6: Comparison between the muon flux obtained by CORSIKA with experimental results obtained by BESS [40] and semianalytical formulae of Nash [37] and Gaisser [35, 39, 41, 42].

azimuthal angle of the muons $\left(0^{\circ}\right.$ for vertical $)$. The parameters $A_{\pi, k}$ are the normalizations of the pion and kaon production spectra. There are several other parameters entering the approximation: the absorption lengths of the primary particle $\lambda_{p}$, of the pions $\lambda_{\pi}$, and kaons $\lambda_{k}$. There is clearly some influence on pion and kaon's production rate [38], but in the present investigation, the values have been fixed along the original proposal [37]. Only the $A_{\pi, k}$ values have been changed in order to adjust the calculated fluxes to the results of the Monte Carlo simulations (see [39]) and to experimental data from the BESS experiment [40].

The spectra of pions and kaons are given by the relation

$$
N\left(p_{\pi, k}\right)=A_{\pi, k} \cdot p_{\pi, k}^{-2.97}
$$

where the factors $A_{\pi}$ and $A_{k}$ have the values $A_{\pi}=0.373$ and $A_{k}=1$.

Semianalytical approaches are able to reproduce globally the results of Monte Carlo simulations and experimental data, and in particular the approach of Judge and Nash does account for muon energies $<10 \mathrm{GeV}$. However, these approaches can be hardly modified in order to take into account also finer effect like the influence of the geomagnetic field. For that detailed Monte Carlo simulations have to be invoked.

The simulation tool COsmic Rays SImulation for KASCADE (CORSIKA) has been originally designed for the four-dimensional simulations of extensive air showers with primary energies around $10^{15} \mathrm{eV}$. The particle transport includes the particle ranges defined by the life time of the particle and its cross-section with air. The density profile of the atmosphere is handled as continuous function thus not sampled in layers of constant density. Ionization losses, multiple scattering, and the deflection in the local magnetic field are considered. The decay of particles is simulated in exact kinematics, and the muon polarization is taken into account. In contrast to other air shower simulations tools CORSIKA offers alternatively six different models for the description of the high energy hadronic interaction and three different models for the description of the low energy hadronic interaction. The threshold between the high and low energy models is set by default to $E_{\mathrm{Lab}}=80 \mathrm{GeV} / \mathrm{n}$.

Figure 6 shows the comparison between the muon flux simulated with CORSIKA compared with the one using semianalytical approaches of Nash and Gaisser and experimental 


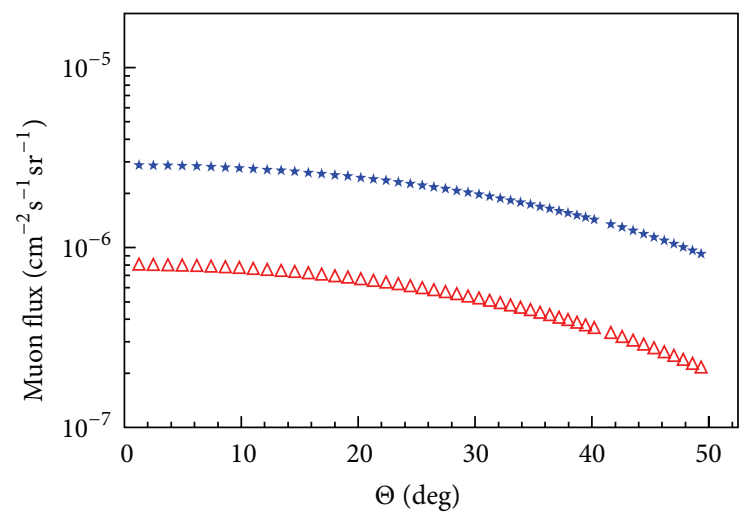

* Muon flux in Unirea mine for the real depth

$\Delta$ Muon flux in Unirea mine for the physical depth

(a)

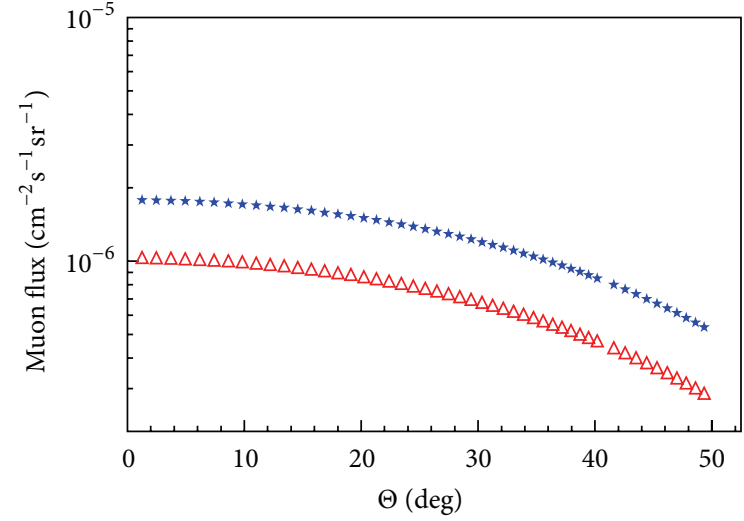

* Muon flux in Cantacuzino-11 mine for the real depth

$\Delta$ Muon flux in Cantacuzino-11 mine for the physical depth

(b)

FIGURE 7: The directional variation of muon flux for the real and theoretical depths of Unirea mine (a) the directional variation of muon flux for the real and theoretical depths of Cantacuzino mine, level 11 (b).

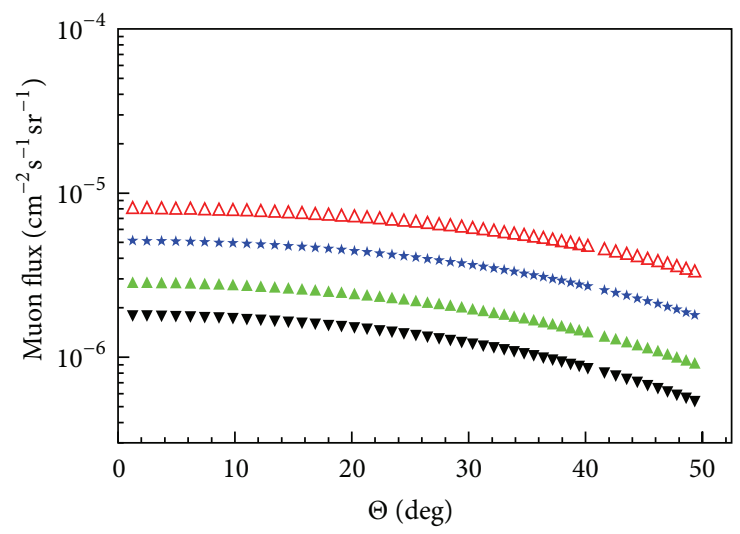

$\Delta$ Muon flux in Mihai mine for the real depth

* Muon flux in Mihai mine-gallery for the real depth

$\Delta$ Muon flux in Unirea mine for the real depth

v Muon flux in Cantacuzino-11 mine for the real depth

FIGURE 8: Simulation of the directional variation of the differential muon flux in the underground of Slanic Prahova salt mines.

data given by BESS experiment. From this picture one can see that the best solution for the muon flux at sea level is represented by CORSIKA simulation code.

In order to perform accurate simulation of the muon flux in the underground, CORSIKA results have been used as input data for MUSIC program and for its extension MUSUN [19].

The directional variation of the muon flux in the underground has been simulated taking into account the angular acceptance of the new detector. The results obtained for the real and for the theoretical depths of Cantacuzino and Unirea mines are displayed in Figure 7. One can observe that important variation of the differential flux for the real and theoretical depths is present. In Figure 8 the directional variation of the muon flux for Unirea, Cantacuzino, and

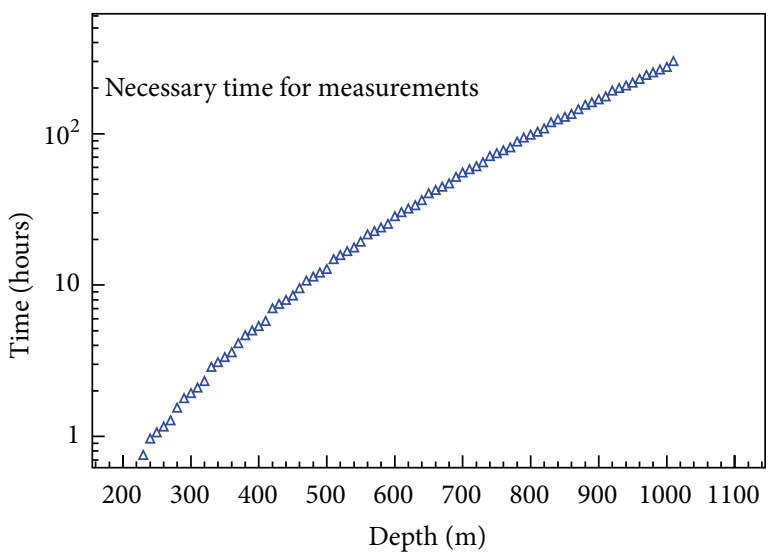

FIgURE 9: Simulation of the muon flux in the underground of Slanic Prahova salt mine.

Mihai mines is presented. The differences are significant and are caused by different depths.

The possibility do detect hidden cavities in the salt dome has been investigated using the MUSIC code. The requested measurement time for the detection of a $10 \mathrm{~m}$ gap starting from $50 \mathrm{~m}$ depth has been calculated taking into account the necessary statistics to obtain valid results. In Figure 9 the variation of the requested measurements time is represented. From this picture one can observe that for depths up to $1000 \mathrm{~m}$ the necessary time is not bigger than 250 hours (aprox. 10 days) which is more than reasonable for the purposes of this project.

\section{Conclusions}

A new detector has been developed which permit directional measurements of the muon flux. The muon flux measurements will be used to study the possibility to detect unknown 
cavities in the old mining sites (e.g., Slanic Prahova). Measurements could be performed on different sites from Europe using the mobile detector, but one should take into account that the for the biggest depths a much longer time is necessary.

A new version of MUSIC computational code has been developed and allows simulation of the muon flux for shallow depths. The code could be adapted for any site and rock composition from the world.

Such muon flux measurements could be also used for geological studies, for example, to explore variations in the rock density above the observation level. The mobility of the detector implies a considerable practical flexibility of using the procedure of measuring muon flux differences for various aspects.

\section{Acknowledgments}

The present work has been possible due to the support of the Romanian Authority for Scientific Research by the projects PN 09370105 and CAPACITATI MARI, 7PM/I/2008, IFINDezvoltarea Infrastructurii de Cercetare. The author thanks Dr. Denis Stanca, Eng. Mihai Niculescu-Oglinzanu, and Eng. Bogdan Cautisanu for their useful advices and help. The author confirms that no financial relationships exist between himself or his relatives and all the companies mentioned in the text exist. The companies have been mentioned only to inform about the structure and the concept of the detection system.

\section{References}

[1] I. Marculet and C. Marculet, Observations on Geomorphological Risk in the Ocna Mures Corridor, Ecological Performance in a Competitive Economy, vol. 2, Bucuresti, Romania, 2011.

[2] O. P. Anchuela, A. P. Juan, A. M. Casas-Sainz, D. Ansón-López, and H. Gil-Garbi, "Actual extension of sinkholes: considerations about geophysical, geomorphological, and field inspection techniques in urban planning projects in the Ebro basin (NE Spain)," Geomorphology, vol. 189, pp. 135-149, 2013.

[3] V. Hess, "Über Beobachtungen der durchdringenden Strahlung bei sieben Freiballonfahrten," Physikalische Zeitschrift, vol. 13, pp. 1084-1091, 1912.

[4] J. Ellis, "Beyond the standard model with the LHC," Nature, vol. 448, no. 7151, pp. 297-301, 2007.

[5] P. K. Grieder, Cosmic Rays at Earth, Researcher's Reference Manual and Data Book, Elsevier, 2001.

[6] A. Haungs, W. D. Apela, J. C. Arteaga et al., "The air-shower experiment KASCADE-grande," Nuclear Physics B, vol. 169, pp. 80-85, 2009.

[7] H. Falcke, W. D. Apel, A. F. Badea et al., "Detection and imaging of atmospheric radio flashes from cosmic ray air showers," Nature, vol. 435, pp. 313-316, 2005.

[8] J. Abraham, P. Abreu, M. Aglietta et al., "Measurement of the energy spectrum of cosmic rays above $10^{18} \mathrm{eV}$ using the Pierre Auger Observatory," Physics Letters B, vol. 685, no. 4-5, pp. 239246, 2010.

[9] K. Shinozaki and M. Teshima, "AGASA results," Nuclear Physics $B$, vol. 136, pp. 18-27, 2004.
[10] J. Wentz, A. F. Badea, A. Bercuci et al., "The relevance of the muon charge ratio for the atmospheric neutrino anomaly," Journal of Physics G, vol. 27, no. 7, p. 1699, 2001.

[11] D. Heck, J. Knapp, J. N. Capdevielle, G. Schatz, and T. Thouw, Report FZKA, 6019 Forschungszentrum Karlsruhe, 1998.

[12] A. Geranios et al., "AIRES EAS simulations for the energy estimation of UHECR," in Proceedings of the 29th International Cosmic Ray Conference, Pune, INDIA, August 2005.

[13] X. Duan et al., "A shannon-runge-kutta-gill method for convection-diffusion equations," Mathematical Problems in Engineering, vol. 2013, Article ID 163734, 5 pages, 2013.

[14] A. Rubbia, "Underground neutrino detectors for particle and astroparticle Science: The Giant Liquid Argon Charge Imaging ExpeRiment (GLACIER)," Journal of Physics, vol. 171, no. 1, Article ID 012020.

[15] P. Schreiner and M. Goodman, "Measurement of the muon charge ratio in MINOS," in Proceedings of the 29th International Cosmic Ray Conference, vol. 9, pp. 97-98, Pune, India, 2005.

[16] L. W. Alvarez, J. A. Anderson, F. El Bedwei et al., "Search for hidden chambers in the pyramids," Science, vol. 167, no. 3919, pp. 832-839, 1970.

[17] R. Alfaro, E. Belmont-Moreno, A. Cervantes et al., "A muon detector to be installed at the Pyramid of the Sun," Revista Mexicana de Fisica, vol. 49, no. 4, pp. 54-59, 2003.

[18] L. Olah, G. G. Barnafoldi, G. Hamar, H. G. Melegh, G. Suranyi, and D. Varga, "Cosmic muon detection for geophysical applications," Advances in High Energy Physics, vol. 2013, Article ID 560192, 7 pages, 2013.

[19] V. Kudryavtsev et al., "Muon simulation codes MUSIC and MUSUN for underground physics," Computer Physics Communications, vol. 180, no. 3, pp. 339-346, 2009.

[20] B. Mitrica et al., "A mobile detector for measurements of the atmospheric muon flux in underground sites," Nuclear Instruments and Methods in Physics Research A, vol. 654, no. 1, pp. 176-183, 2011.

[21] B. Mitrica, D. Stanca, M. Petcu et al., "A mobile detector for muon measurements based on two different techniques," Advances in High Energy Physics, vol. 2013, Article ID 256230, 7 pages, 2013.

[22] S. Agostinelliae, J. Allison, and K. Amako, "Geant 4-a simulation toolkit," Nuclear Instruments and Methods in Physics Research A, vol. 506, Article ID 250303, 2003.

[23] R. Margineanu, C. Simion, S. Bercea et al., "The SlanicPrahova (ROMANIA) underground low-background radiation laboratory," Applied Radiation and Isotopes, vol. 66, no. 10, pp. 1501-1506, 2008.

[24] C. A. Simion, N. Paunescu, N. Mocanu et al., "Ultra low radiation background LSC measurements in a salt mine: a feasibility study," Journal of Labelled Compounds and Radiopharmaceuticals, vol. 53, no. 5-6, pp. 307-311, 2010.

[25] A. Rubbia, "The LAGUNA design study-towards giant liquid based underground detectors for neutrino physics and astrophysics and proton decay searches," Acta Physica Polonica B, vol. 41, no. 7, pp. 1727-1732, 2010.

[26] http://www.isc.kharkov.com/.

[27] A. R. Sterian, "Computer modeling of the coherent optical amplifier and laser systems," in International Conference on Computational Science and Its Applications (ICCSA '07), vol. 4705 of Lecture Notes in Computer Science, pp. 436-449, Kuala Lumpur, Malaysia, August, 2007.

[28] http://www.hamamatsu.com/. 
[29] http://www.caen.it/.

[30] http://www.ni.com/.

[31] M. Bektasoglu, H. Arslan, and D. Stanca, "Simulations of muon flux in slanic salt mine," Advances in High Energy Physics, vol. 2012, Article ID 751762, 8 pages, 2012.

[32] A. Fassò, A. Ferrari, and P. R. Sala, "Electronphoton transport in FLUKA: status," in Proceedings of the MonteCarlo 2000 Conference, A. Kling, F. Barao, M. Nakagawa, L. Tavora, and P. Vaz, Eds., pp. 159-164, Springer, Berlin, Germany, October 2000.

[33] http://www.fluka.org/.

[34] T. K. Gaisser, "Semi-analytic approximations for production of atmospheric muons and neutrinos," Astroparticle Physics, vol. 16, no. 3, pp. 285-294, 2002.

[35] T. K. Gaisser, Cosmic Rays and Particle Physics, Cambridge University Press, Cambridge, UK, 1992.

[36] M. Unger et al., "Results from L3+C," International Journal of Modern Physics A, vol. 20, p. 6928, 2005.

[37] R. J. R. Judge and W. F. Nash, "Measurements on the muon flux at various zenith angles," Il Nuovo Cimento Series 10, vol. 35, no. 4, pp. 999-1024, 1965.

[38] I. M. Brancus, H. Rebele, A. Haungs et al., "WILLI-a scintillator detector setup for studies of the zenith and azimuth variation of charge ratio and flux of atmospheric muons," Nuclear Physics B-Proceedings Supplements, vol. 175-176, pp. 370-373, 2008.

[39] B. Mitrica, "Asymmetry of charge ratio for low energetic muons," AIP Conference Proceedings, vol. 972, pp. 500-504, 2008.

[40] M. Motoki et al., "Precise measurements of atmospheric muon fluxes with the BESS spectrometer," Astroparticle Physics, vol. 19, no. 1, pp. 113-126, 2003.

[41] B. Mitrica et al., "KASCADE-Grande, Forschungszentrum Karlsruhe," Internal Report 2004-01, 2004.

[42] H. Rebel, J. Wentz, I. M. Brancus, O. Sima, B. Mitrica, and G. Toma, "Scientific report on the NATO project," Tech. Rep. Nr.PDD(CP) (PST.CLG, 979737), 2004. 

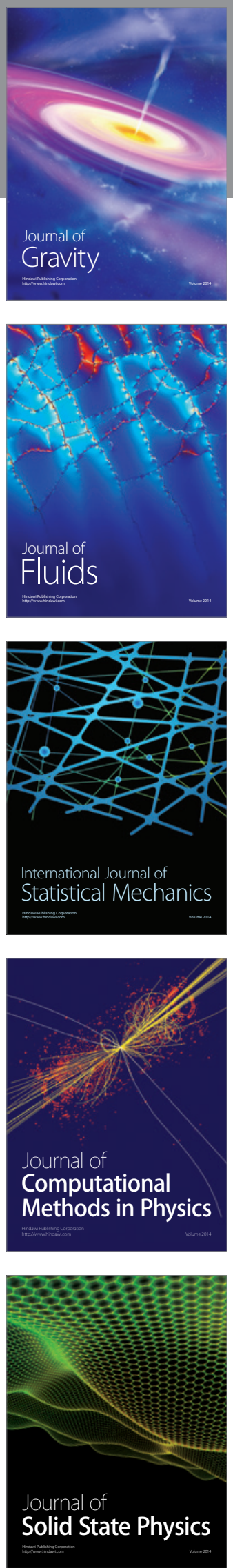

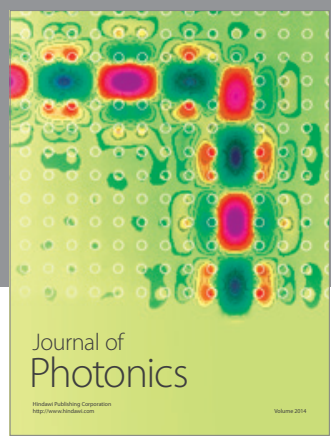

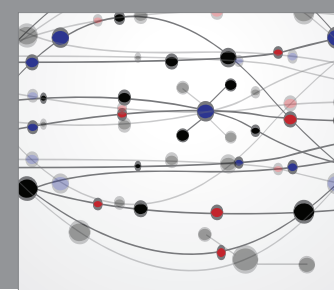

The Scientific World Journal

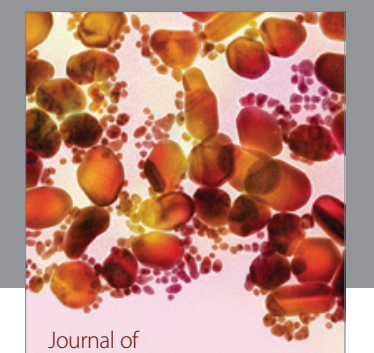

Soft Matter
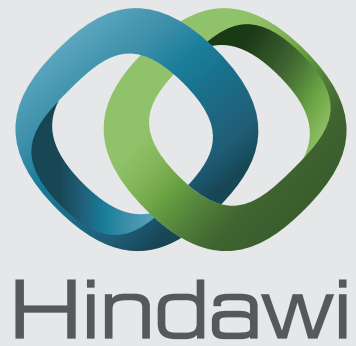

Submit your manuscripts at

http://www.hindawi.com
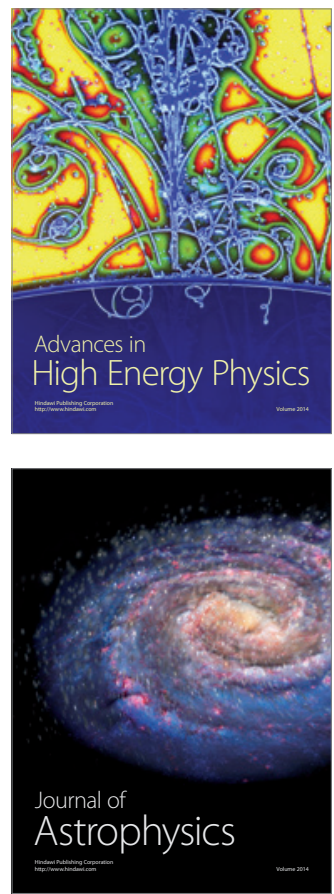
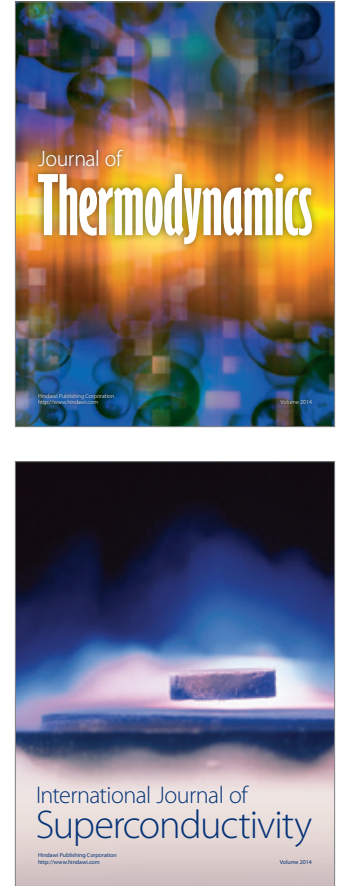
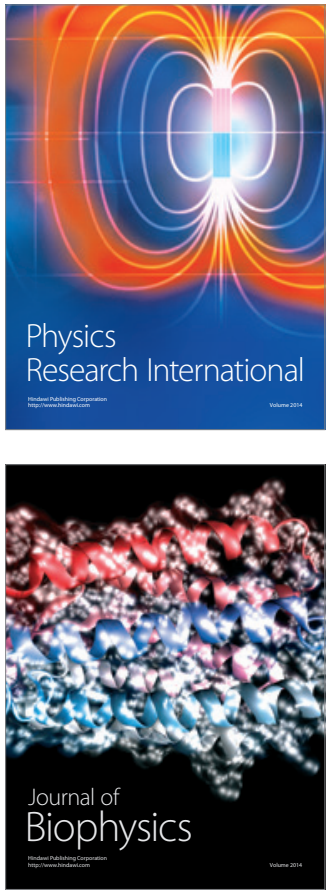
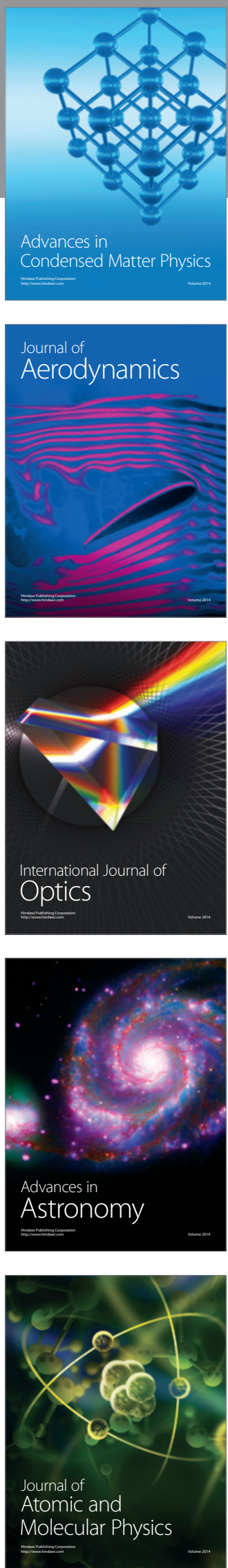